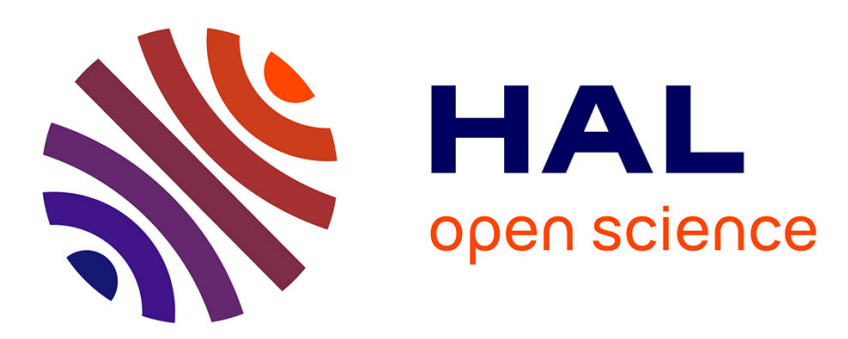

\title{
Electron and Gamma Background in CRESST Detectors
}

R.F. Lang, G. Angloher, M. Bauer, I. Bavykina, A. Bento, A. Brown, C. Bucci, C. Ciemniak, C. Coppi, G. Deuter, et al.

\section{To cite this version:}

R.F. Lang, G. Angloher, M. Bauer, I. Bavykina, A. Bento, et al.. Electron and Gamma Background in CRESST Detectors. Astroparticle Physics, 2009, 32 (6), pp.318. 10.1016/j.astropartphys.2009.09.009 . hal-00510616

\section{HAL Id: hal-00510616 https://hal.science/hal-00510616}

Submitted on 20 Aug 2010

HAL is a multi-disciplinary open access archive for the deposit and dissemination of scientific research documents, whether they are published or not. The documents may come from teaching and research institutions in France or abroad, or from public or private research centers.
L'archive ouverte pluridisciplinaire HAL, est destinée au dépôt et à la diffusion de documents scientifiques de niveau recherche, publiés ou non, émanant des établissements d'enseignement et de recherche français ou étrangers, des laboratoires publics ou privés. 


\section{Accepted Manuscript}

Electron and Gamma Background in CRESST Detectors

R.F. Lang, G. Angloher, M. Bauer, I. Bavykina, A. Bento, A. Brown, C. Bucci, C. Ciemniak, C. Coppi, G. Deuter, F. von Feilitzsch, D. Hauff, S. Henry, P. Huff, J. Imber, S. Ingleby, C. Isaila, J. Jochum, M. Kiefer, M. Kimmerle, H. Kraus, J.-C. Lanfranchi, B. Majorovits, M. Malek, R. McGowan, V.B. Mikhailik, E. Pantic, F. Petricca, S. Pfister, W. Potzel, F. Pröbst, S. Roth, K. Rottler, C. Sailer, K. Schäffner, J. Schmaler, S. Scholl, W. Seidel, L. Stodolsky, A.J.B. Tolhurst, I. Usherov, W. Westphal

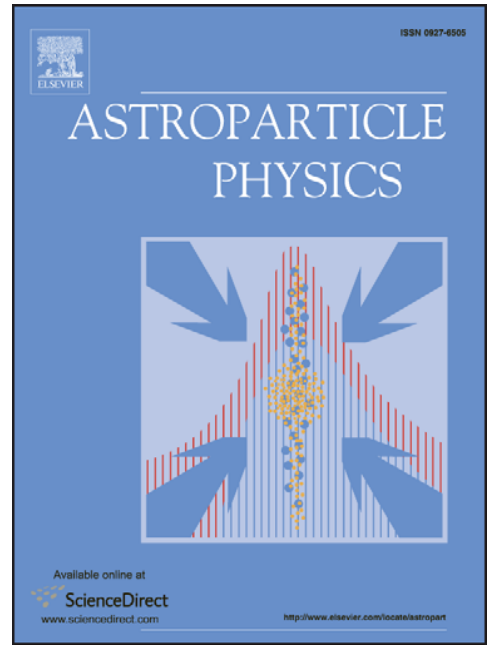

PII: S0927-6505(09)00144-3

DOI: 10.1016/j.astropartphys.2009.09.009

Reference: ASTPHY 1437

To appear in: Astroparticle Physics

Received Date: 25 April 2009

Revised Date: 13 September 2009

Accepted Date: 27 September 2009

Please cite this article as: R.F. Lang, G. Angloher, M. Bauer, I. Bavykina, A. Bento, A. Brown, C. Bucci, C. Ciemniak, C. Coppi, G. Deuter, F. von Feilitzsch, D. Hauff, S. Henry, P. Huff, J. Imber, S. Ingleby, C. Isaila, J. Jochum, M. Kiefer, M. Kimmerle, H. Kraus, J.-C. Lanfranchi, B. Majorovits, M. Malek, R. McGowan, V.B. Mikhailik, E. Pantic, F. Petricca, S. Pfister, W. Potzel, F. Pröbst, S. Roth, K. Rottler, C. Sailer, K. Schäffner, J. Schmaler, S. Scholl, W. Seidel, L. Stodolsky, A.J.B. Tolhurst, I. Usherov, W. Westphal, Electron and Gamma Background in CRESST Detectors, Astroparticle Physics (2009), doi: 10.1016/j.astropartphys.2009.09.009

This is a PDF file of an unedited manuscript that has been accepted for publication. As a service to our customers we are providing this early version of the manuscript. The manuscript will undergo copyediting, typesetting, and review of the resulting proof before it is published in its final form. Please note that during the production process errors may be discovered which could affect the content, and all legal disclaimers that apply to the journal pertain. 


\title{
Electron and Gamma Background in CRESST Detectors
}

R. F. Lang ${ }^{\mathrm{a}}$, G. Angloher ${ }^{\mathrm{a}}$, M. Bauer ${ }^{\mathrm{d}}$, I. Bavykina ${ }^{\mathrm{a}}$, A. Bento ${ }^{\mathrm{a}, \mathrm{f}}$, A. Brown $^{\mathrm{c}}$, C. Bucci ${ }^{\mathrm{e}}$, C. $_{\text {Ciemniak }}^{\mathrm{b}}$, C. Coppi ${ }^{\mathrm{b}}$, G. Deuter ${ }^{\mathrm{d}}$, F. von Feilitzsch ${ }^{\mathrm{b}}$, D. Hauff ${ }^{\mathrm{a}}$, S. Henry ${ }^{\mathrm{c}}$, P. Huff ${ }^{\mathrm{a}}$, J. Imber ${ }^{\mathrm{c}}$, S. Ingleby ${ }^{\mathrm{c}}$,

C. Isaila ${ }^{\mathrm{b}}$, J. Jochum ${ }^{\mathrm{d}}$, M. Kiefer ${ }^{\mathrm{a}}$, M. Kimmerle ${ }^{\mathrm{d}}$, H. Kraus ${ }^{\mathrm{c}}$, J.-C. Lanfranchi ${ }^{\mathrm{b}}$, B. Majorovits ${ }^{\mathrm{a}, \mathrm{c}}$, M. Malek ${ }^{c}$, R. McGowan ${ }^{\mathrm{c}}$, V. B. Mikhailik ${ }^{\mathrm{c}}$, E. Pantic ${ }^{\mathrm{a}}$, F. Petricca ${ }^{\mathrm{a}}$, S. Pfister ${ }^{\mathrm{b}}$, W. Potzel ${ }^{\mathrm{b}}$, F. Pröbst ${ }^{\mathrm{a}}$, S. Roth ${ }^{b}$, K. Rottler ${ }^{d}$, C. Sailer ${ }^{d}$, K. Schäffner ${ }^{a}$, J. Schmaler ${ }^{a}$, S. Scholl ${ }^{d}$, W. Seidel ${ }^{\mathrm{a}}$, L. Stodolsky ${ }^{\mathrm{a}}$,

A. J. B. Tolhurst ${ }^{\mathrm{c}}$, I. Usherov ${ }^{\mathrm{d}}$, W. Westphal ${ }^{\mathrm{b}, \mathrm{g}}$

${ }^{a}$ Max-Planck-Institut für Physik, Föhringer Ring 6, D-80805 München, Germany

${ }^{b}$ Physik-Department E15, Technische Universität München, D-85747 Garching, Germany

${ }^{c}$ Department of Physics, University of Oxford, Oxford OX1 3RH, United Kingdom

${ }^{d}$ Eberhard-Karls-Universität Tübingen, D-72076 Tübingen, Germany

${ }^{e}$ INFN, Laboratori Nazionali del Gran Sasso, I-67010 Assergi, Italy

fon leave from: Departamento de Fisica, Universidade de Coimbra, P3004 516 Coimbra, Portugal

${ }^{g}$ Deceased

\begin{abstract}
The CRESST experiment monitors $300 \mathrm{~g} \mathrm{CaWO}_{4}$ crystals as targets for particle interactions in an ultra low background environment. In this paper, we analyze the background spectra that are recorded by three detectors over many weeks of data taking. Understanding these spectra is mandatory if one wants to further reduce the background level, and allows us to cross-check the calibration of the detectors. We identify a variety of sources, such as intrinsic contaminations due to primordial radioisotopes and cosmogenic activation of the target material. In particular, we detect a $3.6 \mathrm{keV} \mathrm{X}$-ray line from the decay of ${ }^{41} \mathrm{Ca}$ with an activity of $(26 \pm 4) \mu \mathrm{Bq}$, corresponding to a ratio ${ }^{41} \mathrm{Ca} /{ }^{40} \mathrm{Ca}=(2.2 \pm 0.3) \times 10^{-16}$.
\end{abstract}

Key words: CRESST, CaWO 4 , Low Background, Calcium-41, Dark Matter PACS: 29.40.Vj, 95.35.+d

\section{Introduction}

The Cryogenic Rare Event Search with Superconducting Thermometers CRESST [1, 2] aims to detect rare nuclear recoils from elastic scattering of Dark Matter particles [3]. Since less than one such recoil is expected per kilogram of target mass and week of exposure (e.g. [2]), efficient shielding of the target against ambient radioactivity is mandatory.

To this end, the CRESST experiment is located in the Laboratori Nazionali del Gran Sasso under an average of $1400 \mathrm{~m}$ of rock overburden, where the cosmic muon flux is reduced by about six orders of magnitude with respect to the surface. The target is surrounded (from the outside to the inside) by $45 \mathrm{~cm}$ of polyethylene to moderate the neutron flux, a radon box that is constantly flushed with nitrogen gas to reduce the concentration of radioactive

Email address: rafael.lang@mpp.mpg.de (R. F. Lang) Preprint submitted to Astroparticle Physics radon and its daughters inside the shielding, as well as $20 \mathrm{~cm}$ of lead and $14 \mathrm{~cm}$ of pure copper against the external gamma background [2]. In addition, the expected WIMP-induced nuclear recoil signal can be discriminated from the remaining electron and gamma background utilizing the scintillation light of the $\mathrm{CaWO}_{4}$ crystals [1]. The understanding of the remaining radioactive background present in the experiment prior to this discrimination is subject of this paper. It demonstrates the excellent performance of CRESST detectors and is a necessary step to improve the sensitivity of the experiment to rare processes.

\section{Setup}

The target consists of individual $300 \mathrm{~g} \mathrm{CaWO}_{4}$ crystals that are operated as calorimeters [1]. Up to 33 such detectors can be arranged in a com-

October 6, 2009 
pact layout [2]. To enhance the temperature signal following an interaction, the crystals are cooled to $\sim 15 \mathrm{mK}$ where heat capacities are low. The high frequency phonons that are created in interactions in the crystal are collected by a thermometer, which is a thin tungsten film that is evaporated directly onto the crystals. This film becomes superconducting at these temperatures and is stabilized in its transition to the superconducting state. A small temperature rise results in an increase of resistance that is measured with a SQUID-based readout circuit [4].

A resistive heater structure is attached to each thermometer. This structure is used to provide a constant heating bias as well as for periodic injection of additional heat pulses. Each such injected heat pulse briefly drives the thermometer out of its transition. The amplitude response of the thermometer to the injected heat is controlled to stay at a constant value with small adjustments to the heating bias by means of a feedback system. In this way the operating point of the thermometer is stabilized over several months to within the required $\mu K$ precision [1]. This can also be seen from the sharpness of gamma lines discussed here.

\section{Pulse Height Evaluation}

Our thermometers yield rather slow pulses with rise times of about $1 \mathrm{~ms}$. These pulses are digitized with a time base of $40 \mu \mathrm{s}$. 4096 such samples comprise a record, of which the first 1024 samples are taken from the time before the trigger to define the baseline of the pulse.

A template pulse is fitted to the record in order to evaluate the amplitude of the pulse as a measure of the deposited energy. This template is an average of appropriate pulses, often obtained from a gamma calibration line. Since the SQUIDs only measure relative changes in current, the baseline needs to be defined individually for each recorded pulse. Hence, the free parameters in the fit are the level of the baseline, the onset of the pulse, and its amplitude.

The pulse samples the transition curve of the thermometer from its superconducting to the normal conducting state. For interactions with recoil energies below $\sim 200 \mathrm{keV}$, the resultant pulse generally samples only the linear region of the thermometer's transition curve between the superconducting and normal conducting state. For interactions with larger energies, the pulse samples a region of the transition curve that is non-linear, distorting the resulting pulse shape. Therefore, in the template pulse fit, only those parts of the pulse are included that show a linear behavior. This linearizes the energy response of the fitted amplitudes over a wide energy range, even up to the $\mathrm{MeV}$ range [5].

\section{Energy Calibration}

To reach the cryogenic temperatures, the detectors are enclosed by a fivefold thermal shield made from copper. Calibration sources are placed outside this shield, which has a total thickness of $12 \mathrm{~mm}$ of copper. This requires gamma energies $\gtrsim 100 \mathrm{keV}$ in order to penetrate this barrier. Hence, a method is required to take the energy calibration to lower energies.

To this end, additional heater pulses corresponding to a number of fixed energies are injected into the heater structure every 30 seconds. The pulse height of the response pulse from the thermometer is also evaluated with a template fit. Non-linearities are due to the shape of the transition curve and due to the readout circuit. They manifest themselves in the amplitude of the response pulse, but, for given amplitude, not in its shape. Therefore, the amplitudes of the response pulses give the correct energy assessment even if there are small differences in the pulse shape of heater response pulse and particle pulse.

The heater pulses are calibrated at relatively high energies, typically with the $122 \mathrm{keV}$ or $136 \mathrm{keV}$ line from a ${ }^{57} \mathrm{Co}$ source. The amplitude response of the thermometer as a function of energy injected into the heater can then be fitted with a low-order polynomial function. Figure 1 depicts this procedure for the calibration of particle pulses in the complete energy range covered by the heater pulses. In the following we identify a variety of lines at the expected energies, thus validating our calibration procedure.

\section{Calibration Spectrum}

Figure 2 shows a typical spectrum from a ${ }^{57} \mathrm{Co}$ calibration. The two strong peaks at $122 \mathrm{keV}$ and $136 \mathrm{keV}$ originate from gamma transitions in the ${ }^{57}$ Co source. They show pronounced shoulders towards lower energies, which originate from photons that are Compton scattered in the copper thermal shields and the detector support structures that surround the detectors. 


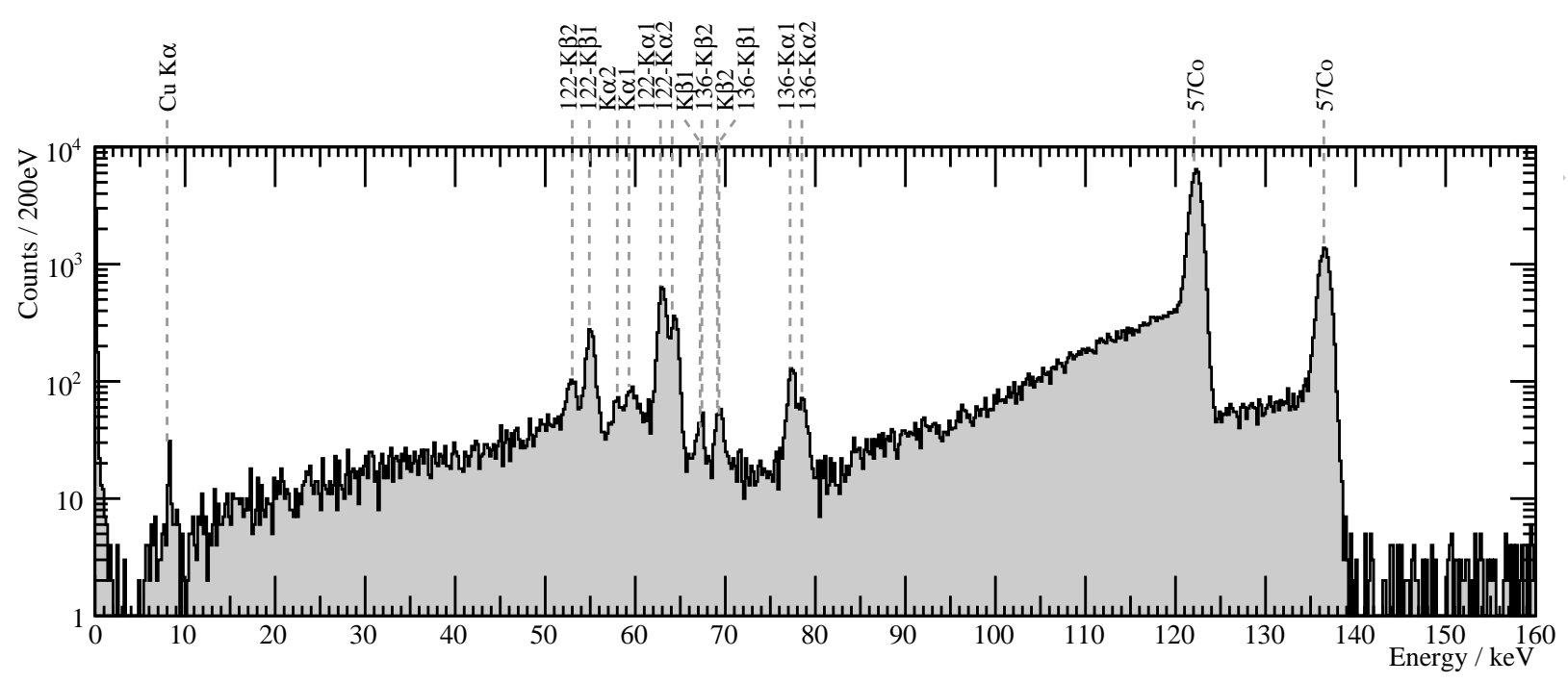

Figure 2: Energy spectrum from a high statistics ${ }^{57}$ Co calibration (detector DAISY, run 31). The relative strengths of escape peaks to direct gamma lines depend on the position of the crystal, the source, as well as the position of neighbouring crystals and the surrounding cryostat and is in agreement with simulations.

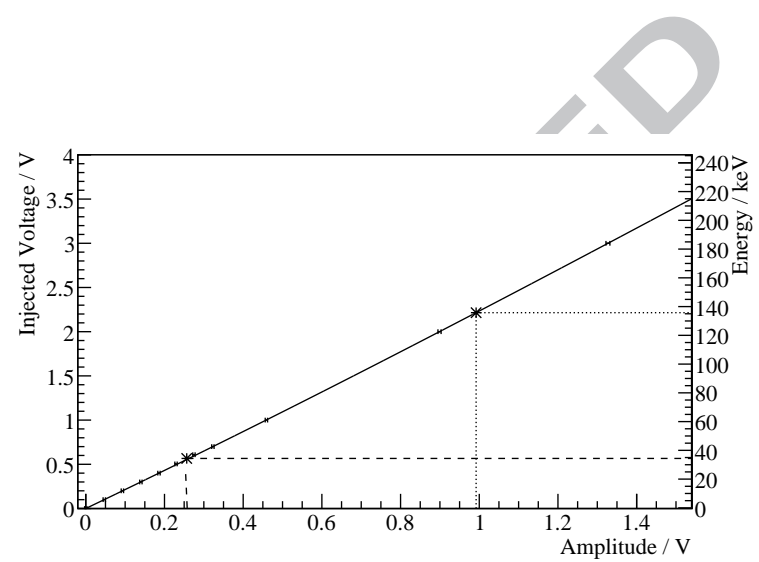

Figure 1: Calibration at low energies with heater pulses of various discrete energies. Fitting a low order polynomial to the thermometer response (small points, including error bars) gives the transfer function (solid line) between injected voltage (scale on the left) and fitted amplitude (scale on the bottom). The $136 \mathrm{keV}$ peak gives a calibration point (dotted line) for the injected energy (scale on the right). Then, for each particle pulse, the particle's energy is found by evaluating the transfer function at the fitted amplitude (dashed line).
A gamma can interact in the crystal via the photoelectric effect, ejecting an electron from its shell. The X-ray produced in the subsequent recombination may escape from the crystal. In this case, we measure the energy of the original gamma minus the energy of the X-ray (escape peak). If the escaping X-ray reaches a neighboring crystal, its energy will be measured there. In figure 2 we observe a set of peaks in the energy range between $50 \mathrm{keV}$ and $80 \mathrm{keV}$, originating from the dominant $K_{\alpha 1}, K_{\alpha 2}$, $K_{\beta 1} / K_{\beta 3}$ and $K_{\beta 2}$ escape lines of tungsten X-rays. The origin of the various observed lines are labeled in the figure. In addition, a line is visible at $8 \mathrm{keV}$ from copper fluorescence due to interactions in the copper surrounding the cryostat (see section 6.6).

The calibration spectrum allows confirmation of our energy calibration method. This is shown in table 1, where measured energies are compared to the literature values [6] for a few lines. The values agree within the resolution of our detectors. Even the extrapolation of our energy calibration to high energies is validated by the $692 \mathrm{keV}$ line from ${ }^{57} \mathrm{Co}$, which is reconstructed only about $1 \%$ below the expected value, namely at $682 \mathrm{keV}$ with a resolution of $3 \mathrm{keV}(1 \sigma)$.

\section{Background Spectra}

In the following, spectra from exposure to background radiation alone are analyzed, from one de- 


\begin{tabular}{lrrr}
\hline $\begin{array}{l}\text { line } \\
\text { origin }\end{array}$ & $\begin{array}{r}\text { literature } \\
\text { value }\end{array}$ & $\begin{array}{r}\text { reconstructed } \\
\text { energy }\end{array}$ & $\begin{array}{r}\text { resolution } \\
(1 \sigma)\end{array}$ \\
\hline $\mathrm{Cu} \mathrm{K}_{\alpha}$ & $8.0 \mathrm{keV}$ & $8.3 \mathrm{keV}$ & $0.1 \mathrm{keV}$ \\
$122 \mathrm{keV}-\mathrm{K}_{\beta 1}$ & $54.9 \mathrm{keV}$ & $55.0 \mathrm{keV}$ & $0.4 \mathrm{keV}$ \\
$\mathrm{W} \mathrm{K}_{\alpha 1}$ & $59.3 \mathrm{keV}$ & $59.6 \mathrm{keV}$ & $0.5 \mathrm{keV}$ \\
$122 \mathrm{keV}-\mathrm{K}_{\alpha 1}$ & $62.8 \mathrm{keV}$ & $63.0 \mathrm{keV}$ & $0.3 \mathrm{keV}$ \\
${ }^{57} \mathrm{Co}$ & $122.1 \mathrm{keV}$ & $122.2 \mathrm{keV}$ & $0.6 \mathrm{keV}$ \\
${ }^{57} \mathrm{Co}$ & $136.5 \mathrm{keV}$ & $136.5 \mathrm{keV}$ & $0.6 \mathrm{keV}$ \\
\hline
\end{tabular}

Table 1: Literature values, reconstructed energies and resolution of a few lines observed in the cobalt calibration. All values agree and validate our energy calibration procedure. The energy resolution is seen to be better than $1 \mathrm{keV}$.

tector during the prototyping phase of the experiment [1] (figures 3,4) and two detectors during the commissioning phase [2] (figures 5, 8 and 6). All these crystals were grown by the General Physics Institute, Moscow, Russia, but in different batches.

\subsection{Strontium and Yttrium}

Powders containing calcium are difficult to purify from strontium since both elements belong to the same chemical group. Thus, strontium can easily remain in our $\mathrm{CaWO}_{4}$ crystals during the Czochralski growth process. Indeed this is the case, as can be seen in figure 3 , where we show the spectrum of one crystal up to $1 \mathrm{MeV}$. We observe a continuous background which can be attributed to the beta decay of ${ }^{90} \mathrm{Sr}$ (with an endpoint energy of $546 \mathrm{keV}$ ) together with the subsequent beta decay of ${ }^{90} \mathrm{Y}(2282 \mathrm{keV}$ endpoint energy).

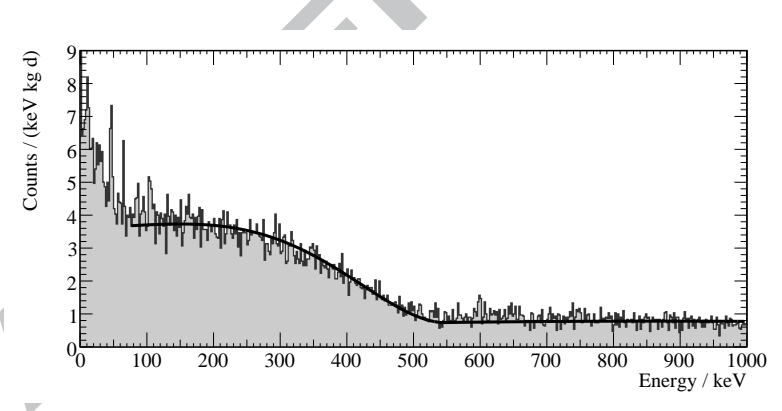

Figure 3: Background spectrum observed with one crystal $(m=306.8 \mathrm{~g})$ from an exposure to background radiation of $15.00 \mathrm{~kg}$ d early 2004 (detector DAISY, run 28). The black line is a fit above $80 \mathrm{keV}$ of a parametrization of the ${ }^{90} \mathrm{Sr} /{ }^{90} \mathrm{Y}$ beta spectrum to the data.

A fit above $80 \mathrm{keV}$ to a parametrization of the expected beta spectrum from ${ }^{90} \mathrm{Sr}$ in equilibrium with ${ }^{90} \mathrm{Y}$ [7] can be seen to describe the data in this energy range. From the fit we deduce an activity of
$(4.37 \pm 0.15) \mathrm{mBq}$ of ${ }^{90} \mathrm{Sr}$ for this crystal. In addition to this common and continuous background, the three crystals show quite different features in the energy range below $100 \mathrm{keV}$.

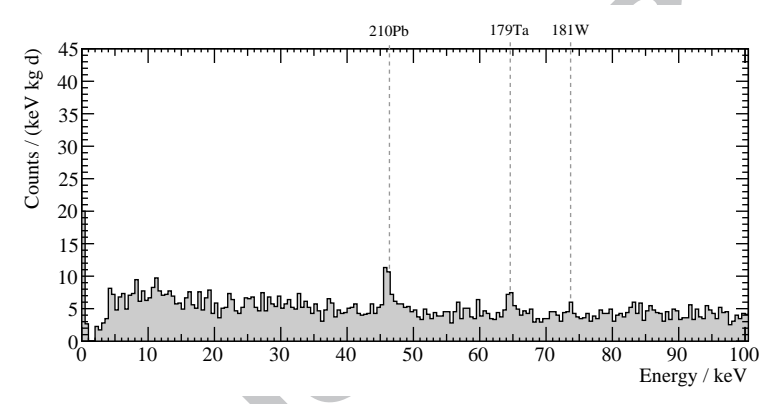

Figure 4: Zoom on the energy region below $100 \mathrm{keV}$ of the spectrum shown in figure 3.

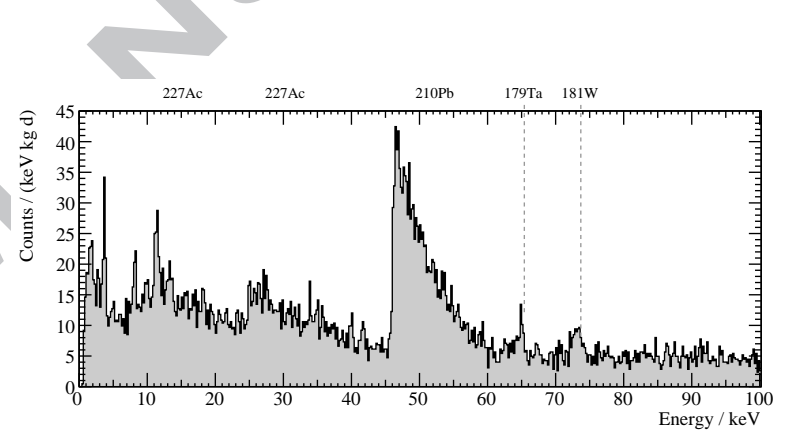

Figure 5: Background spectrum of a second crystal $(m=$ $305.5 \mathrm{~g}$ ) from an exposure of $21.20 \mathrm{~kg} \mathrm{~d}$ in 2007 (detector VERENA, run 30).

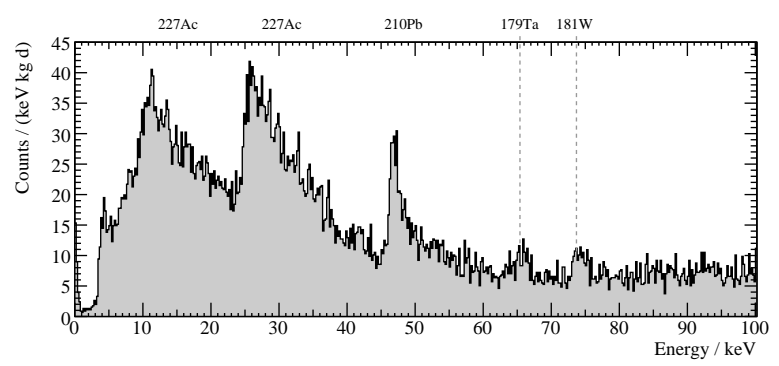

Figure 6: Background spectrum recorded in 2007 from a third detector $(m=307.0 \mathrm{~g})$ with an exposure of $22.81 \mathrm{~kg} \mathrm{~d}$ (detector Zora, run 30).

\subsection{Lead}

Isotopes from natural decay chains are present in the crystals and can be identified by their alpha decays [5]. A step in the natural decay of ${ }^{238} \mathrm{U}$ is ${ }^{210} \mathrm{~Pb}$, which beta-decays into ${ }^{210} \mathrm{Bi}$ with an endpoint energy of $63.5 \mathrm{keV}$. In $84 \%$ of these decays 
a $46.5 \mathrm{keV}$ gamma is emitted. If the decays happen in the vicinity of our crystals, we observe a line at $46.5 \mathrm{keV}$ (see figure 4 ), which reflects an external activity of $(31 \pm 5) \mu \mathrm{Bq}$. If, on the other hand, ${ }^{210} \mathrm{~Pb}$ is a contamination intrinsic to the crystal, our calorimetric measurement gives the energy of the gamma plus that of the emitted electron. Hence, we observe a beta spectrum starting at $46.5 \mathrm{keV}$ that extends up to $63.5 \mathrm{keV}$, as can prominently be seen in figure 5 . The activity of this decay due to sources both internal and external to the crystal is much higher, namely $(672 \pm 28) \mu \mathrm{Bq}$. Figure 6 also shows this lead feature for the third crystal with an activity of $(241 \pm 25) \mu \mathrm{Bq}$.

\subsection{Actinium}

A similar case is that of ${ }^{227} \mathrm{Ac}$, a step in the natural decay of ${ }^{235} \mathrm{U} .{ }^{227}$ Ac beta-decays with an endpoint energy of $44.8 \mathrm{keV}$ into ${ }^{227} \mathrm{Th}$, where two excited levels may lead to the emission of $24.5 \mathrm{keV}$ and $9.3 \mathrm{keV}$ gammas. This results in two beta spectra, one starting at $9.3 \mathrm{keV}$, and one at $24.5 \mathrm{keV}$, as can prominently be seen in figure 6 but also in figure 5 . This contamination is not observable in the spectrum shown in figure 4 , which indicates that this detector contains less impurities from the decay of ${ }^{235} \mathrm{U}$.

\subsection{Activated Tungsten}

If the raw material or the crystals themselves are exposed to cosmic radiation, the tungsten in the $\mathrm{CaWO}_{4}$ crystals can be activated. A possible channel is ${ }^{182} \mathrm{~W}(p, \alpha)^{179} \mathrm{Ta} .{ }^{179} \mathrm{Ta}$ then decays via electron capture with a half-life of 1.8 years into ${ }^{179} \mathrm{Hf}$. The energy signature of this decay in our crystals is the binding energy of the captured electron, mostly from the K-shell, which is $E_{\mathrm{K}, \mathrm{Hf}}=65.4 \mathrm{keV}$. The activation via ${ }^{183} \mathrm{~W}(p, t)$ results in ${ }^{181} \mathrm{~W}$. This decays with a half-life of 121 days via electron capture into an excited ${ }^{181}$ Ta nucleus, which de-excites with emission of a $6.2 \mathrm{keV}$ gamma. Hence, the dominant energy signature of this decay is the binding energy of a tantalum K-shell electron, $E_{\mathrm{K}, \mathrm{Ta}}=67.4 \mathrm{keV}$, plus the energy of the gamma, $6.2 \mathrm{keV}$, adding up to $73.7 \mathrm{keV}$.

Lines at these energies can be seen in all spectra. For the crystal of which the spectrum is shown in figure 4, a measurement with a similar exposure to background radiation, about three months earlier, is available [8]. The crystal was stored underground in between these two measurements, so no re-activation could occur. This allows us to extract the ${ }^{179} \mathrm{Ta}$ and ${ }^{181} \mathrm{~W}$ activities from two measurements. A comparison to the expected decay times is shown in figure 7 and confirms the origin of these lines.

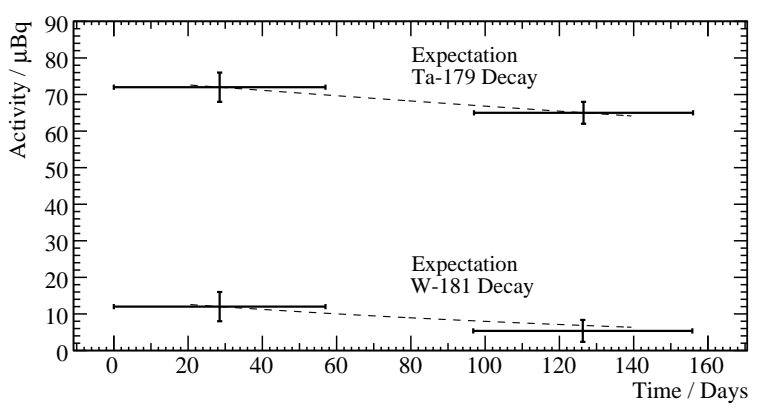

Figure 7: Two measurements of the ${ }^{181} \mathrm{~W}$ and ${ }^{179} \mathrm{Ta}$ activities in one crystal during two runs with similar exposure to background radiation. The expectations from decays with $T_{1 / 2}=121 \mathrm{~d}$ and $T_{1 / 2}=665 \mathrm{~d}$ are drawn as dashed lines and seen to be consistent with the measurement. Error bars are uncertainties of the fit to the spectral lines (each containing tens of counts) and the running time of the respective measurements (detector DAISY, run 27 and run 28).

\subsection{Activated Calcium}

Capture of thermal neutrons from cosmic rays can activate calcium present in the $\mathrm{CaWO}_{4}$ crystals. ${ }^{41} \mathrm{Ca}$ has a half-life of $10^{5}$ years and decays via electron-capture to ${ }^{41} \mathrm{~K}$. The $\mathrm{K}$-shell binding energy of potassium is only $3.61 \mathrm{keV}$, but our detectors are capable of detecting such low energies. Figure 8 shows again the spectrum of figure 5 but for lower energies and with a finer binning. We observe a prominent line at $(3.71 \pm 0.02) \mathrm{keV}$ (mean and error from the fit of a Gaussian). This is only $3 \%$ above the expected energy from ${ }^{41} \mathrm{Ca}$, which we therefore attribute to this line. The width of this line is only $(130 \pm 19) \mathrm{eV}$, consistent with the calibration. For illustration, figure 9 shows a typical pulse at this low energy, still clearly visible above baseline noise.

From the observed activity of $(26 \pm 4) \mu \mathrm{Bq}$ we can readily calculate that there are $(1.4 \pm 0.2) \times 10^{8}$ ${ }^{41} \mathrm{Ca}$ atoms present in the crystal, only a fraction of $(2.2 \pm 0.3) \times 10^{-16}$ of all calcium atoms. The level of activity is consistent with expectations for raw materials mined from a few meters below surface. Our measurement is an order of magnitude more accurate than other measurements of this fraction, which are typically carried out by accelerator mass 


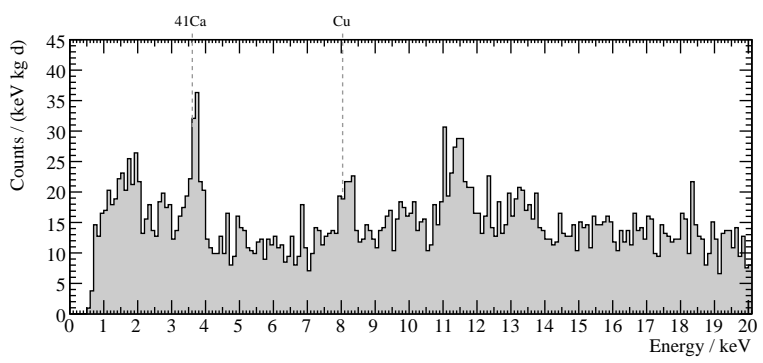

Figure 8: Low-energy part of the spectrum shown in figure 5. In addition to the lines from ${ }^{41} \mathrm{Ca}$ and copper fluorescence there is a line at $\sim 11.5 \mathrm{keV}$ which so far could not be identified in a consistent way (detector VERENA, run 30).

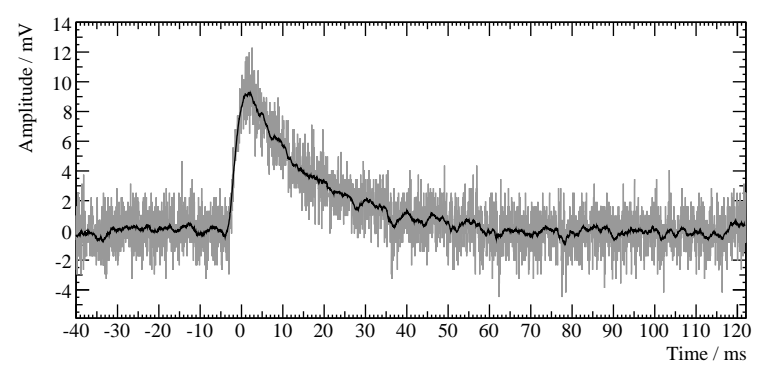

Figure 9: A typical pulse with an energy of $3.7 \mathrm{keV}$ (grey), and its 50-sample ( $2 \mathrm{~ms}$ ) moving average (black).

spectrometry (AMS) [9, 10, 11]. Our present sensitivity is in the $10^{-17}$ range even with $\mathrm{CaWO}_{4}$ crystals that are not optimized for this purpose. This demonstrates the extreme sensitivity of our detectors for rare decays and opens new possibilities for radioactive dating using ${ }^{41} \mathrm{Ca}[12]$. In fact, having an in situ calibration line at these low energies is an advantage for the direct Dark Matter search, since it independently confirms our energy calibration procedure in this otherwise inaccessible region.

\subsection{Copper Fluorescence}

Another line, seen at $(8.17 \pm 0.04) \mathrm{keV}$ in figure 8, can be attributed to copper fluorescence. This originates from gammas or electrons that eject a photoelectron from the copper of the cryostat that surrounds the detectors, resulting in a copper X-ray absorbed in our crystals. Our energy calibration puts the line only $2 \%$ higher than the expected $8.04 \mathrm{keV}$ from the literature. This is consistent with the slight energy overestimate seen when identifying the ${ }^{41} \mathrm{Ca}$ spectral line, again confirming its origin.

\subsection{Lutetium}

All single crystal spectra above $100 \mathrm{keV}$ are rather featureless. Yet, two crystals were operated simultaneously and in close vicinity during the commissioning of the setup. This allows us to search for coincident events, of which a spectrum is shown in figure 10 .

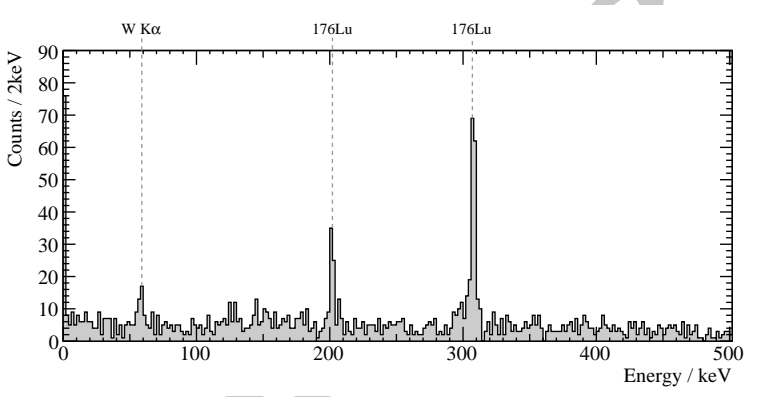

Figure 10: Spectrum of coincident events. To increase the counting statistics, the spectra of the two individual detectors were added to produce this figure. Without the coincidence requirement, the added spectrum would have a count rate of $\mathcal{O}(500)$ counts per $2 \mathrm{keV}$ bin (detectors VERENA and ZORA, run 30).

At $59 \mathrm{keV}$, tungsten $\mathrm{K}_{\alpha}$ escape events can be seen. In addition, two lines at $202 \mathrm{keV}$ and $307 \mathrm{keV}$ become very prominent, and we attribute those to a contamination with lutetium intrinsic to the crystals. ${ }^{176} \mathrm{Lu}$ beta-decays to ${ }^{176} \mathrm{Hf}$ with a total decay energy of $1191.7 \mathrm{keV}$. Each decay produces a gamma cascade with gamma energies of $306.8 \mathrm{keV}$, $201.8 \mathrm{keV}$ and $88.3 \mathrm{keV}$. In particular the higher energy gammas may escape from one crystal and hit the other. Hence, for a coincident $306.8 \mathrm{keV}$ event observed in one detector, the other crystal will mostly absorb the other two gammas, plus the energy of the beta electron. This explains the structure seen in the scatter plot of figure 11, showing the energies of coincident events in each detector. We observe a beta spectrum starting at $201.8 \mathrm{keV}+88.3 \mathrm{keV}=290.1 \mathrm{keV}$ for most events at $306.8 \mathrm{keV}$ in the other detector (lines marked (a) and (b) in figure 11). Figure 12 shows the energy spectrum of these events in one detector, tagged by an energy of $306.8 \mathrm{keV}$ in the other one, in agreement with the expectation from the lutetium beta spectrum.

\section{Conclusions}

The excellent energy resolution of less than $1 \mathrm{keV}$ over the full energy range used in the Dark Matter search $[1,2]$ allows us to identify a variety of different background sources in the CRESST experiment. Since the CRESST experiment can discrimi- 


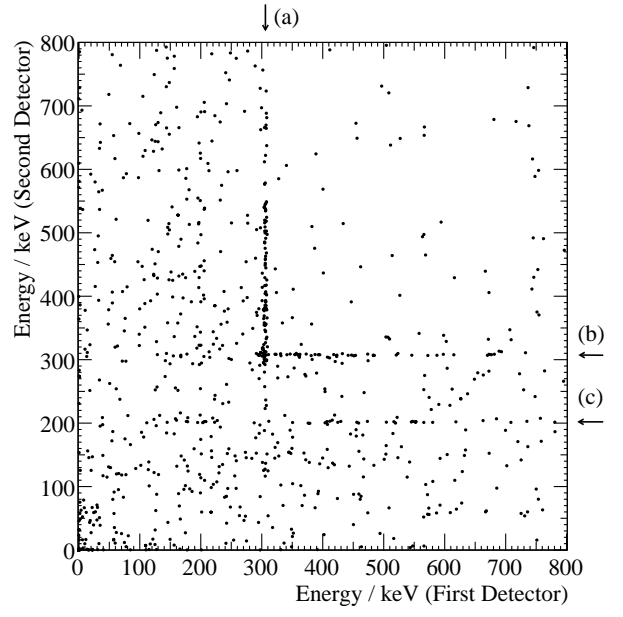

Figure 11: Scatter plot of coincident events in two detectors operated during commissioning. The vertical line (a) going up from $290 \mathrm{keV}$ is due to lutetium decaying in the second detector, in coincidence with the $307 \mathrm{keV}$ gamma escaped to the first detector. The horizontal line (b) is due to the same process with detectors reversed, at $307 \mathrm{keV}$ in the second detector and starting at $290 \mathrm{keV}$ in the first one. At (c) we observe a hint of a horizontal line starting at $395 \mathrm{keV}$ due to lutetium decaying in the first detector with the energy of an escaped $202 \mathrm{keV}$ gamma in the second detector.

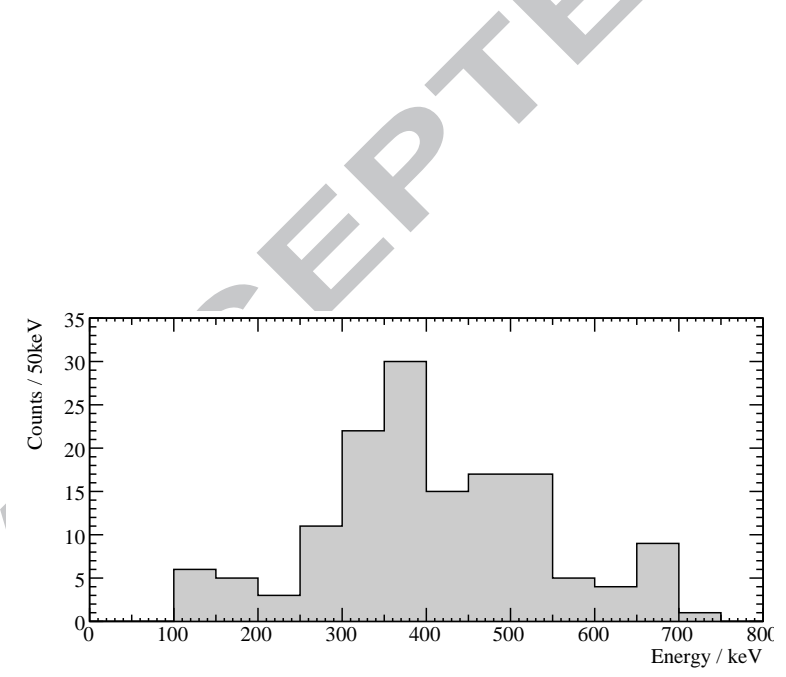

Figure 12: Histogram of all coincident events in one detector that appear at $(307 \pm 3) \mathrm{keV}$ in the respective other detector: The ${ }^{176} \mathrm{Lu}$ beta spectrum starting at $290 \mathrm{keV}$ is visible despite the low statistics. nate nuclear recoil signal from electron recoil background based on the light output, the presence of these features does not compromise the search for Dark Matter, yet allows an audit of the performance of the experiment. The energy resolution $(1 \sigma)$ is $130 \mathrm{eV}$ at $3.6 \mathrm{keV}$, and the observed sharpness of the lines proves the high stability of the detectors during the background measurements running for several months. The observed line from activated calcium provides an independent in situ energy calibration even below the energy range used for the Dark Matter search, and in addition proves the high sensitivity of the experiment to rare decays.

\section{Acknowledgments}

R. F. L. acknowledges useful discussions with G. Korschinek on ${ }^{41} \mathrm{Ca}$. This work was partially supported by funds of the DFG (SFB 375 and Transregio 27 "Neutrinos and Beyond"), the $\mathrm{Mu}-$ nich Cluster of Excellence ("Origin and Structure of the Universe"), the EU networks for Cryogenic Detectors (ERB-FMRXCT980167) and for Applied Cryogenic Detectors (HPRN-CT2002-00322), and the Maier-Leibnitz-Laboratorium (Garching). Support was provided by the Science and Technology Facilities Council.

\section{References}

[1] G. Angloher et al. (The CRESST Collaboration), Astroparticle Physics 23 (2005) 325, arXiv:astro-ph/0408006.

[2] G. Angloher et al. (The CRESST Collaboration), Astroparticle Physics 31 (2009) 270, arXiv:0809.1829.

[3] G. Jungman, M. Kamionkowski and K. Griest, Physics Reports 267 (1996) 195, arXiv: hep-ph/9506380.

[4] W. Seidel et al., Physics Letters B 236 (1990) 483.

[5] C. Cozzini et al., Physical Review C 70 (2004) 064606, arXiv:nucl-ex/0408006.

[6] R. B. Firestone et al., Table of Isotopes (CD ROM Edition), 1st ed., John Wiley \& Sons, New York, 1996.

[7] K. Kossert, private communication, 2009.

[8] B. Majorovits et al. (2006), in: The Identification of Dark Matter, Proceedings of the Sixth International Workshop, edited by G. Fanourakis, M. Axenides and J. Vergados, 192, World Scientific.

[9] S. Merchel et al., Nuclear Instruments and Methods B (2009), to be published.

[10] D. Fink et al., Nuclear Instruments and Methods B 47 (1990) 79.

[11] W. Henning et al., Nuclear Instruments and Methods A 257 (1987) 60.

[12] G. M. Raisbeck and F. Yiou, Nature 277 (1979) 42. 